\title{
Selection of hyperadherent mutants in Pseudomonas putida biofilms
}

\section{Correspondence \\ Manuel Espinosa-Urgel \\ manuel.espinosa@eez.csic.es}

Received 21 December 2011

Revised 27 April 2011

Accepted 17 May 2011

\author{
Fátima Yousef-Coronado, ${ }^{1,2}$ María Isabel Soriano, ${ }^{1}$ Liang Yang, ${ }^{2}$ \\ Søren Molin ${ }^{2}$ and Manuel Espinosa-Urgel ${ }^{1}$
}

\author{
${ }^{1}$ Department of Environmental Protection, Estación Experimental del Zaidín, Consejo Superior de \\ Investigaciones Científicas (CSIC), Granada, Spain \\ ${ }^{2}$ Center for Systems Microbiology, Technical University of Denmark, Lyngby, Denmark
}

\begin{abstract}
A number of genetic determinants required for bacterial colonization of solid surfaces and biofilm formation have been identified in different micro-organisms. There are fewer accounts of mutations that favour the transition to a sessile mode of life. Here we report the isolation of random transposon Pseudomonas putida KT2440 mutants showing increased biofilm formation, and the detailed characterization of one of them. This mutant exhibits a complex phenotype, including altered colony morphology, increased production of extracellular polymeric substances and enhanced swarming motility, along with the formation of denser and more complex biofilms than the parental strain. Sequence analysis revealed that the pleiotropic phenotype exhibited by the mutant resulted from the accumulation of two mutations: a transposon insertion, which disrupted a predicted outer membrane lipoprotein, and a point mutation in lapG, a gene involved in the turnover of the large adhesin LapA. The contribution of each alteration to the phenotype and the possibility that prolonged sessile growth results in the selection of hyperadherent mutants are discussed.
\end{abstract}

\section{INTRODUCTION}

The development of a multicellular community associated with a surface and surrounded by an exopolymeric matrix, referred to as a biofilm, is common to a variety of bacteria under different environmental conditions. Biofilm formation has received increasing attention due to its importance in medicine, since biofilm populations are considered relevant to chronic infection, and are more resistant to the action of antibiotics and biocidals than planktonic populations (Anderson \& O'Toole, 2008; Høiby et al., 2010). Biofilm development is also relevant in industrial settings and in the design of bioreactors (Nicolella et al., 2000; Singh et al., 2006). Genetic determinants that play a role in biofilm formation have been unravelled in different bacterial species, and environmental and cellular signals that influence this process have also been described. These include iron availability, quorum sensing, and the intracellular secondary messenger cyclic di-GMP (Banin et al., 2006; Patriquin et al., 2008; Ueda \& Wood, 2009; Coenye, 2010). Changes in the levels of cyclic di-GMP correlate with phenotypic changes associated with virulence, motility, colony morphology, production of exopolysaccharides,

Abbreviations: CLSM, confocal laser-scanning microscopy; EPS, extracellular polymeric substances.

A supplementary figure, showing P. putida KT2440 and ibi-626 lapG sequences, is available with the online version of this paper. and the transition between planktonic and sessile lifestyles (Hengge, 2009; Römling \& Simm, 2009, and references therein). Changes in the expression of different functions are also associated with one or the other lifestyle; in Pseudomonas aeruginosa and Pseudomonas putida, for example, differential expression of flagellar components has been observed when comparing planktonic and biofilm populations, and even during the various stages of biofilm development (Sauer \& Camper, 2001; Sauer et al., 2002; Toutain et al., 2007). In the former organism, swarming motility and surface attachment are inversely regulated through a pathway that involves BifA, a cyclic-di-GMP phosphodiesterase, and the membrane protein SadC (Merritt et al., 2007; Kuchma et al., 2007).

Biofilm formation under various experimental settings follows a sequence of events that begins with a fraction of the bacterial population attaching to the solid surface and settling on it, while the rest remain as planktonic cells or attach and immediately detach from the surface, in what has been called 'reversible attachment' (Monds \& O'Toole, 2009). Our own observations of adhesion of the plantbeneficial bacterium $P$. putida KT2440 to plant seeds indicate that only a small percentage of the population initiates attachment to the surface (Espinosa-Urgel et al., 2000; Yousef-Coronado et al., 2008). This leaves open the possibility that in the planktonic population a subpopulation specialized in surface colonization arises, and therefore 
it might be possible to isolate mutants locked in a biofilmprone genetic programme. Mutations that cause an increase in the intracellular levels of cyclic di-GMP have been shown to enhance biofilm formation and reduce detachment from the surface in different micro-organisms (Kuchma et al., 2010; Gjermansen et al., 2006). In Escherichia coli, inactivation of $c y s E$, the gene encoding serine acetyltransferase, which converts serine into $O$-acetyl-L-serine, a molecule that may act as an extracellular signal, results in accelerated biofilm formation (Sturgill et al., 2004). In $P$. putida, lapG, a gene that causes reduced detachment when disrupted, has been described (Gjermansen et al., 2010). Recently (Newell et al., 2011), it has been proven that LapG has proteolytic activity towards LapA, the major adhesin of Pseudomonas fluorescens and P. putida, and seems to be a key element in the turnover of LapA on the bacterial surface in response to environmental signals. In this work we report the isolation of $P$. putida mutants that show increased biofilm formation, and the characterization of mutations that alter the normal attachment/detachment dynamics of this bacterium.

\section{METHODS}

Bacterial strains, plasmids and growth conditions. $P$. putida KT2440, a plasmid-free derivative of $P$. putida mt-2 (Regenhardt et al., 2002), was used in this study. Mutants ibi-626 and EU9 were obtained as described below. E. coli JM109 was used as host for cloning experiments, with plasmid pBBR1-MCS5 as cloning vector (Kovach et al., 1995). E. coli donor and helper strains used for random transposon mutagenesis with mini- $\operatorname{Tn} 5[\mathrm{Km} 1]$ have been described elsewhere (de Lorenzo et al., 1990). Derivatives of KT2440 and ibi-626 carrying $g f p$ in single copy in the chromosome were obtained using mini-Tn7, as described by Koch et al. (2001).

Unless otherwise stated, cultures were grown in liquid Luria-Bertani (LB) medium (Lennox, 1955) or 1:10 LB, and on LB plates with $1.5 \%$ agar, at $30{ }^{\circ} \mathrm{C}$ (P. putida) or $37{ }^{\circ} \mathrm{C}$ (E. coli). When appropriate, antibiotics were used at the following concentrations (in $\mu \mathrm{g}^{-1}$ ): ampicillin, 100; chloramphenicol, 30; kanamycin, 50; gentamicin, 10 and 100 for E. coli and P. putida, respectively.

Random transposon mutagenesis and selection strategy for hyperadherent mutants. Transposon mutagenesis with mini-Tn5 was done by triparental conjugation using the protocol previously described (Espinosa-Urgel et al., 2000). Kanamycin-resistant clones were selected in minimal M9 medium with citrate as carbon source. For the selection of mutants with increased attachment capacity, the pool of transposon mutants was collected and $\sim 10^{8}$ cells were inoculated in $3 \mathrm{ml}$ liquid LB medium and grown in borosilicate glass tubes under orbital rotation. After $2 \mathrm{~h}$ of growth, the medium was removed to discard planktonic cells, the tubes were washed with M9, and $3 \mathrm{ml}$ fresh LB was added. The same procedure was repeated three times. In this way, enrichment in mutants having increased attachment was expected. Finally, attached bacteria were allowed to grow for $3 \mathrm{~h}$ and removed from the surface by vortexing for $30 \mathrm{~s}$ in the presence of glass beads ( $3 \mathrm{~mm}$ diameter), followed by plating on selective medium.

Cloning of olpA and construction of olpA deletion mutant EU9. A $1019 \mathrm{bp}$ fragment containing the $\operatorname{lp} A$ gene, encoding a putative outer membrane lipoprotein (see Results), and its upstream region, were PCR-amplified and cloned in pBBR-MCS5, yielding plasmid
pFY1. The insert was sequenced to ensure the absence of mutations. Plasmid pFY1 was used to construct an olpA mutant by replacement of an internal $500 \mathrm{bp}$ fragment with a kanamycin-resistance cassette from p34S-Km3 (Dennis \& Zylstra, 1998). The olpA:: Km fragment was then cloned into pUC19. The resulting plasmid, pFY3, was electroporated into $P$. putida KT2440, and kanamycin-resistant clones were selected. Since the vector does not replicate in this organism, these clones were the result of either the integration of the plasmid into the chromosome by a single recombination event or the replacement of olpA by double recombination. The latter were selected after screening by PCR amplification. One clone (EU9) was chosen, and the corresponding chromosomal region was sequenced to confirm the mutation.

Swarming motility, biofilm formation and biofilm stability assays. Swarming motility was tested on $0.5 \%$ peptone glycerol (PG) agar plates incubated at $25^{\circ} \mathrm{C}$, as previously described (Matilla et al., 2007). Biofilm formation was examined during growth in polystyrene microtitre plates (Sterilin) or in borosilicate glass tubes, as described previously (Yousef-Coronado et al., 2008). Biomass attached to the surface was visually inspected and quantified by staining with crystal violet, followed by solubilization of the dye with $70 \%$ ethanol and measurement of $A_{580}$ (O'Toole \& Kolter, 1998). Biofilm stability after enzymic treatment was tested by incubating biofilms grown in microtitre plates (after $4 \mathrm{~h}$ of growth) with DNase $\left(0.1 \mathrm{mg} \mathrm{ml}^{-1}\right)$, cellulase $\left(5 \mathrm{mg} \mathrm{ml}^{-1}\right)$ or proteinase $\mathrm{K}\left(0.05 \mathrm{mg} \mathrm{ml}^{-1}\right)$ for 15 or $80 \mathrm{~min}$, and then staining and quantifying the attached biomass as described above. Data are given as the percentage of biomass retained in the biofilm with respect to untreated controls. Analysis of variance (ANOVA) and Student's $t$ test were used for statistical analysis.

A modification of the method described by Gjermansen et al. (2006) was used for biofilm formation under flow conditions, using LB diluted $1: 10$ as growth medium. Biofilms were grown at $30{ }^{\circ} \mathrm{C}$ in three-channel flow chambers (BioCentrum-DTU, Technical University of Denmark), using a Watson-Marlow 205S peristaltic pump. Overnight cultures were diluted to $\mathrm{OD}_{600} 0.5$, and $300 \mu \mathrm{l}$ was injected into the flow chamber. The flow was turned off during the first hour to allow initial attachment of the cells. The flow was then turned on and kept at a constant rate of $3 \mathrm{ml} \mathrm{h}^{-1}$ (laminar flow conditions). Biofilm structures were visualized at different times of development with a Nikon $\mathrm{C} 1$ confocal laser-scanning microscope. Images were analysed with Imaris software (Bitplane), and biofilm parameters were calculated using COMSTAT (Heydorn et al., 2000).

Microscopy. Colony morphology was visualized and photographed using a Euromex inverted microscope. For transmission electron microscopy, bacteria were recovered from exponentially growing cultures $\left(\mathrm{OD}_{660} 0.9\right)$ and adsorbed on Formvar-coated grids, stained with $1 \%$ phosphotungstic acid for $30 \mathrm{~s}$ and air-dried. The grids were observed under a JEOL JEM-1011 transmission electron microscope at $80 \mathrm{kV}$. Images were obtained using an SIS Megaview III capture system and analySIS docu software. High-resolution scanning electron microscopy of biofilm-grown bacteria was performed at the Microscopy Service of Granada University with a Zeiss LEO Gemini 1530.

Molecular biology techniques. Plasmid and chromosomal DNA extraction, electrophoresis and other molecular techniques were performed following standard procedures. For Southern hybridization, a DIG DNA Labeling and Detection kit (Roche) was used. PCR amplification was done using the Expand High Fidelity PCR system (Roche). Restriction enzymes and DNA ligase were used as indicated by the manufacturers (New England Biolabs and Roche).

Isolation and quantification of extracellular polymeric substances (EPS). The method described by Cérantola et al. (2000) was 
used to extract the extracellular matrix components from biofilmgrown cultures. Essentially, biofilms were recovered after growth in LB and homogenized in PBS, and biomass was quantified for normalization by measuring turbidity at $660 \mathrm{~nm}$. Cells were then removed by centrifugation at $10000 \mathrm{~g}$, and the supernatant was precipitated by adding six volumes of $95 \%$ ethanol followed by centrifugation at $12000 \mathrm{~g}\left(30 \mathrm{~min}, 4{ }^{\circ} \mathrm{C}\right)$. The resulting pellet was resuspended in distilled water, dialysed overnight and lyophilized. The presence of DNA and proteins was analysed by electrophoresis and quantified using an ND-1000 spectrophotometer (NanoDrop). Total sugars were quantified using the method described by Altman et al. (1987).

Whole-genome sequencing and analysis. Sequencing of the complete genome of ibi-626 was done by BaseClear BV (Leiden, The Netherlands) using a Roche Illumina Genome Analyzer II. Chromosomal DNA (20 $\mu \mathrm{g})$ was used as template, and 13152665 reads with a mean length of 58 bases were obtained, resulting in a total of 762854570 bases ( 120-fold coverage for the $6.2 \mathrm{Mb}$ genome of KT2440). Mapping of the sequence data was performed using CLCbio Genomic Workbench version 3.7. De novo assemblies were used for comparison with the reference genome sequence of KT2440 in collaboration with Bio-Iliberis R\&D. Potential discrepancies were reassessed by PCR amplification and sequencing of the corresponding region in the wild-type and mutant strains.

\section{RESULTS}

\section{Selection of $\boldsymbol{P}$. putida transposon mutants with increased biofilm initiation capacity}

A screen was carried out to identify P. putida mutants that showed increased biofilm formation, with a focus on the early stages of the bacteria-surface interaction. Random transposon mutagenesis was performed on $P$. putida KT2440 using mini-Tn5[Km1] (de Lorenzo et al., 1990). The resulting kanamycin-resistant clones were pooled, and approximately $10^{7}$ cells were inoculated into liquid LB medium. An enrichment method was designed to identify hyperadherent mutants, consisting of successive rounds of growth in liquid LB, with early removal of planktonic cells and addition of fresh medium, leaving only the initial colonizers of the tube surface, as detailed in Methods. After such rounds of enrichment, bacteria attached to the surface were recovered. Dilutions were plated, and 1920 individual clones were subsequently assayed for biofilm formation in microtitre plates. Clones showing significantly increased attached biomass after $3 \mathrm{~h}$ of growth (termed ibi mutants, for increased biofilm initiation) were chosen for further analysis. Insertion of a single copy of mini-Tn5 was checked by Southern hybridization, using the kanamycinresistance gene as a probe. Clones in which more than one band was observed were discarded. The insertion sites of the minitransposon were determined in the remaining mutants by arbitrarily primed PCR and sequencing, as described in Methods. Mutant ibi-206 was affected in PP_5007, encoding an ancillary protein involved in accumulation of polyhydroxyalkanoate $(\mathrm{PAH})$ granules. $\mathrm{PAH}$ biosynthesis is differentially regulated in biofilm and planktonic cells of $P$. aeruginosa PAO1, and PAH-deficient mutants have been reported to display increased alginate production, associated with changes in biofilm structure and morphology (Pham et al., 2004; Campisano et al., 2008). The gene disrupted in ibi-815 corresponded to putA, encoding proline dehydrogenase. This enzyme catalyses the two-step conversion of proline into glutamic acid, and has a regulatory role in its own expression, as well as in that of the proline transporter $p u t P$; it also plays a role in redox signalling (Vílchez et al., 2000; Fernández-Piñar et al., 2008).

In the remaining four mutants, arbitrary PCR and DNA sequencing placed the insertion of mini-Tn5 in the same position in locus PP_0116. This gene encodes a 235 aa protein highly conserved in all Pseudomonas species sequenced so far. It shows the distinctive features of outer membrane lipoproteins (Yamaguchi et al., 1988; Terada et al., 2001), with a 20 aa signal sequence that is predicted to be cleaved, leaving an $\mathrm{N}$-terminal cysteine, typically involved in lipid binding, followed by a serine residue (MKPFASRYLLVAAFSLILAA/CS). We therefore termed the gene olpA (for outer membrane lipoprotein A). One of these mutants (ibi-626) was chosen for further characterization.

\section{ibi-626 forms thicker and structurally more complex biofilms than KT2440}

Biofilm formation by strain ibi-626 was analysed under different experimental conditions. As shown in Fig. 1, the mutant formed thick biofilms on glass when grown in LB under rotation, leaving the liquid medium almost clear after $8 \mathrm{~h}$, and remaining attached to the surface after $24 \mathrm{~h}$, in contrast to wild-type cells, which were for the most part detached after this period. Quantitative analysis of biofilm development during growth under static conditions was performed in microtitre plates by staining with crystal violet and measuring absorbance. The results confirmed the increased attachment and reduced detachment phenotype of ibi-626 (Fig. 1).

Biofilm development was then analysed in flow chambers by confocal laser-scanning microscopy (CLSM), with KT2440 and ibi-626 derivatives harbouring the $g f p$ gene in single copy in the chromosome. While the wild-type strain formed relatively flat, unstructured biofilms, biofilms of the mutant were not only significantly thicker but also structurally different, with large protrusions and finger-like extensions visible after $6 \mathrm{~h}$ of growth (Fig. 2). Quantitative analysis of biofilm features using the COMSTAT software (Heydorn et al., 2000) showed significant differences in biofilm parameters, including biomass, surface coverage and mean thickness at all times tested (Table 1).

\section{The mutation in olpA is only partially responsible for the phenotype of ibi-626}

To confirm the role of olpA, the wild-type gene was PCRamplified and cloned into pBBR1-MCS5, as described in Methods. The resulting plasmid, $\mathrm{pFY} 1$, was introduced into 


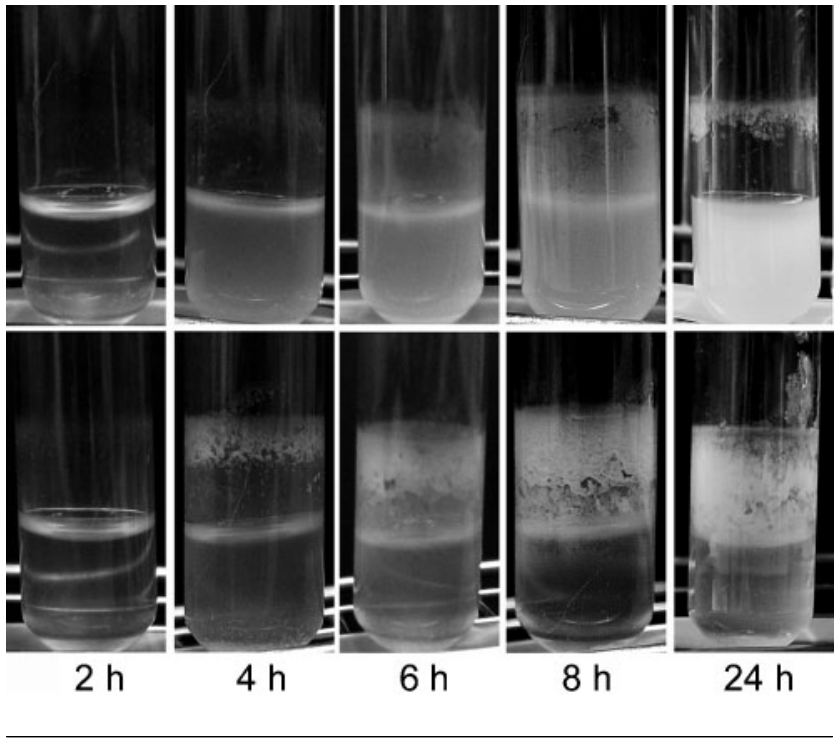

Fig. 1. Attachment of KT2440 (top row) and ibi-626 (bottom row) to glass tubes during growth in LB under orbital aeration.

ibi-626, and the phenotype of the complemented strain was tested. Intriguingly, the intact olpA gene in multicopy did not restore normal biofilm formation kinetics. Thus, ibi626 harbouring pFY1 still formed thicker biofilms than the wild-type (not shown). This could indicate either that the

(a)
KT2440
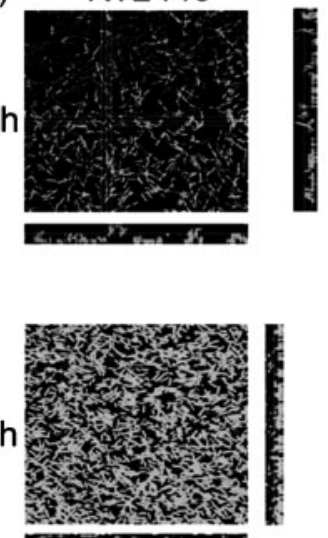

(b)

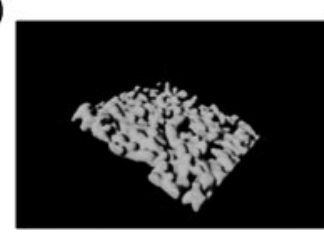

ibi-626
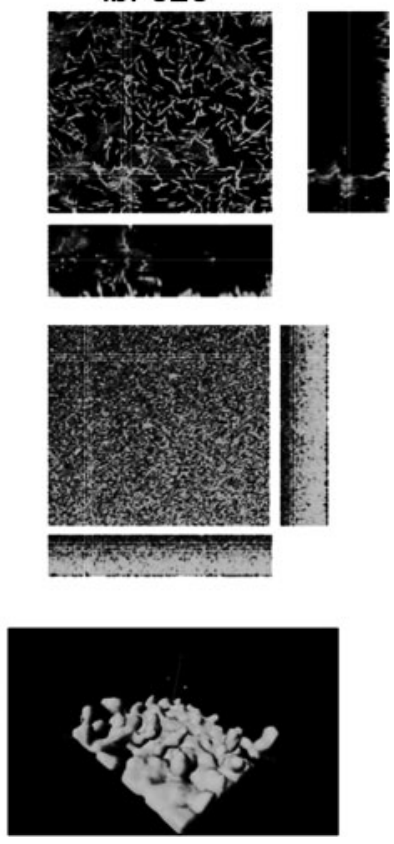

Fig. 2. Top view and sections (a), and $3 D$ reconstructions (b), of biofilm structures formed under flow conditions by KT2440 and ibi-626. CLSM images obtained after 6 and $24 \mathrm{~h}$ of growth were processed with Imaris 3.0 software (Bitplane).
Table 1. COMSTAT analysis of biofilm parameters of KT2440 and ibi-626 under flow conditions in one-tenth strength LB

\begin{tabular}{|lrcc|}
\hline Parameter & Time & KT2440 & ibi-626 \\
\hline Biomass $\left(\mu \mathrm{m}^{3} \mu \mathrm{m}^{-2}\right)$ & $3 \mathrm{~h}$ & $0.670 \pm 0.24$ & $1.485 \pm 0.76$ \\
& $6 \mathrm{~h}$ & $1.093 \pm 0.55$ & $2.169 \pm 1.26$ \\
$24 \mathrm{~h}$ & $3.719 \pm 2.24$ & $20.736 \pm 1.59$ \\
Mean diffusion distance $(\mu \mathrm{m})$ & $3 \mathrm{~h}$ & $0.079 \pm 0.02$ & $0.119 \pm 0.06$ \\
& $6 \mathrm{~h}$ & $0.077 \pm 0.03$ & $0.098 \pm 0.07$ \\
& $24 \mathrm{~h}$ & $0.364 \pm 0.17$ & $4.236 \pm 1.55$ \\
Mean thickness $(\mu \mathrm{m})$ & $3 \mathrm{~h}$ & $0.417 \pm 0.18$ & $1.198 \pm 0.61$ \\
& $6 \mathrm{~h}$ & $0.85 \pm 0.52$ & $2.524 \pm 1.40$ \\
Substratum coverage $(\mu \mathrm{m})$ & $24 \mathrm{~h}$ & $3.997 \pm 2.79$ & $20.321 \pm 1.91$ \\
& $3 \mathrm{~h}$ & $0.263 \pm 0.08$ & $0.384 \pm 0.19$ \\
$6 \mathrm{~h}$ & $0.365 \pm 0.11$ & $0.423 \pm 0.168$ \\
& $24 \mathrm{~h}$ & $0.62 \pm 0.11$ & $0.96 \pm 0.07$ \\
\hline
\end{tabular}

mutation in olpA had a dominant character or that a secondary, spontaneous mutation was responsible for the increased attachment. The fact that the complemented strain showed an intermediate phenotype in terms of swarming motility (see below) suggested a cumulative effect of the olpA mutation and an additional mutation, leading to the pleiotropic effects observed in ibi-626. To test this hypothesis, a null olpA mutant was generated by replacement of the gene with a kanamycin-resistance cassette, as described in Methods. The resulting strain, EU9, did show increased attachment, but only at early time points; further biofilm development and detachment

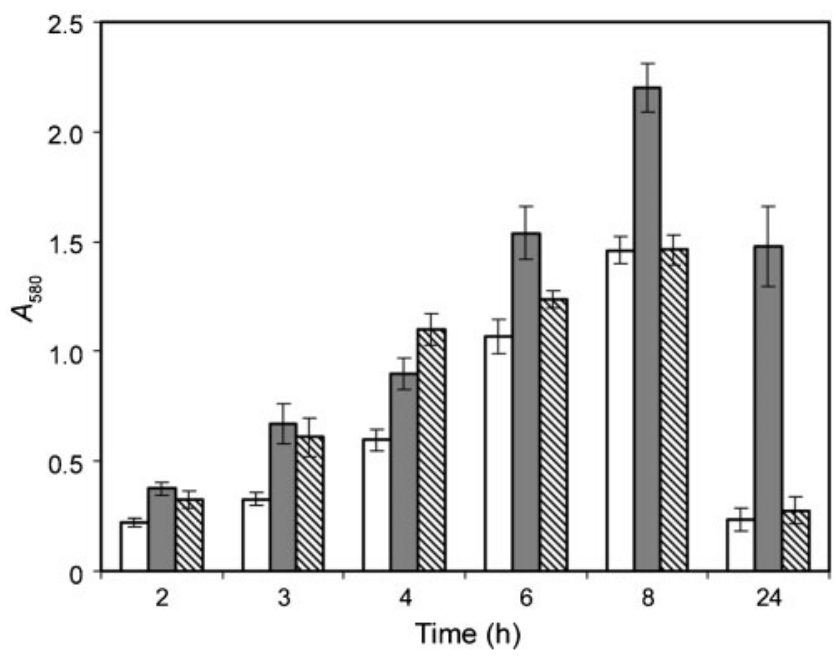

Fig. 3. Quantitative analysis of biofilm formation by KT2440 (white bars), ibi-626 (grey bars) and EU9 (hatched bars) during growth in LB in microtitre plates. Attached biomass was quantified after staining with crystal violet as described in Methods. Data are mean \pm SD from three independent experiments (four replicates per experiment). Differences were significant $(P=0.05)$ between EU9 and KT2440 at 3 and $4 \mathrm{~h}$ of biofilm growth, and between ibi-626 and KT2440 at all time points. 
kinetics were similar to those of the wild-type strain (Fig. 3 ), indicating that the hyperadherent character of ibi-626 is not solely due to the disruption of olpA, and that an additional mutation might be present.

\section{Complete sequencing of ibi-626 reveals a point mutation that affects lapG}

The genome of mutant ibi-626 was fully sequenced and compared with the reference genome of KT2440 (Nelson et al., 2002) to define potential mutations that could contribute to the phenotype of this strain. Several apparent discrepancies with respect to the reference sequence were checked by PCR amplification and sequencing of the corresponding region in ibi-626 and our laboratory KT2440 strain. Most were found to be sequencing inaccuracies or assembly gaps. A single-base insertion present in both strains with respect to the reference sequence was detected in locus PP_3932, yielding a protein longer than annotated in the databases but identical in length to homologous proteins from other Pseudomonas species (data not shown). However, deletion of a base pair within the coding region corresponding to locus PP_0164, 15 bp downstream from the initiation codon, was found in ibi-626, while in KT2440 the coding sequence was identical to the published reference sequence. This change causes a frameshift and early translation stop in the mutant (Supplementary Fig. S1). In both KT2440 and ibi-626, a single base pair upstream of this locus is missing with respect to the published sequence. PP_0164 corresponds to lapG, a gene that has been proposed to act as a signal transducer in biofilm formation (Gjermansen et al., 2005), and more recently has been shown to encode a cysteine protease that affects the function of LapA in P. fluorescens (Newell et al., 2011), the main adhesin of this bacterium

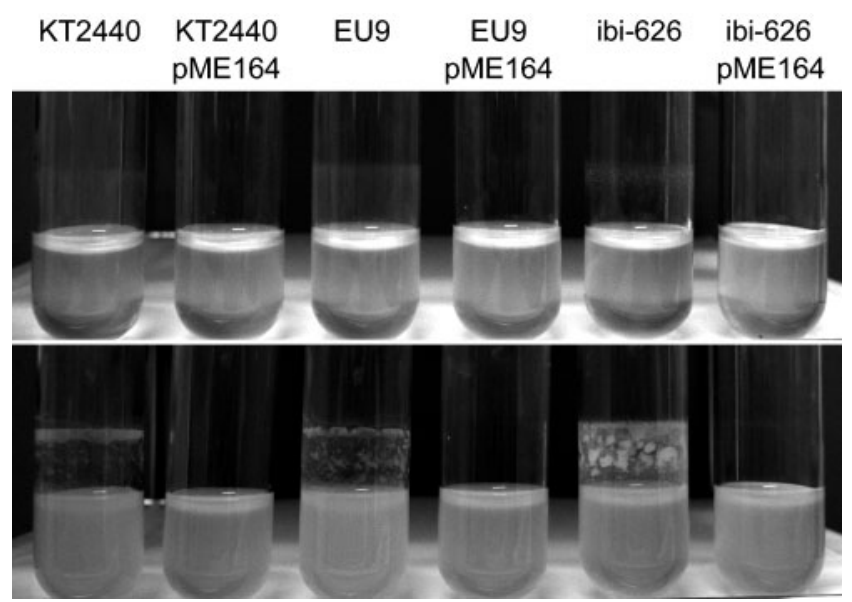

Fig. 4. The presence of lapG in multicopy inhibits biofilm formation. Bacterial attachment was followed during growth in glass tubes under orbital rotation. Images correspond to $3 \mathrm{~h}$ (top row) and $6 \mathrm{~h}$ (bottom row) of growth. and of P. putida (Hinsa et al., 2003; Yousef-Coronado et al., 2008). Transposon mutants in which lapG is disrupted have been shown to be defective in detachment from biofilms, an effect that is compensated when lapA is

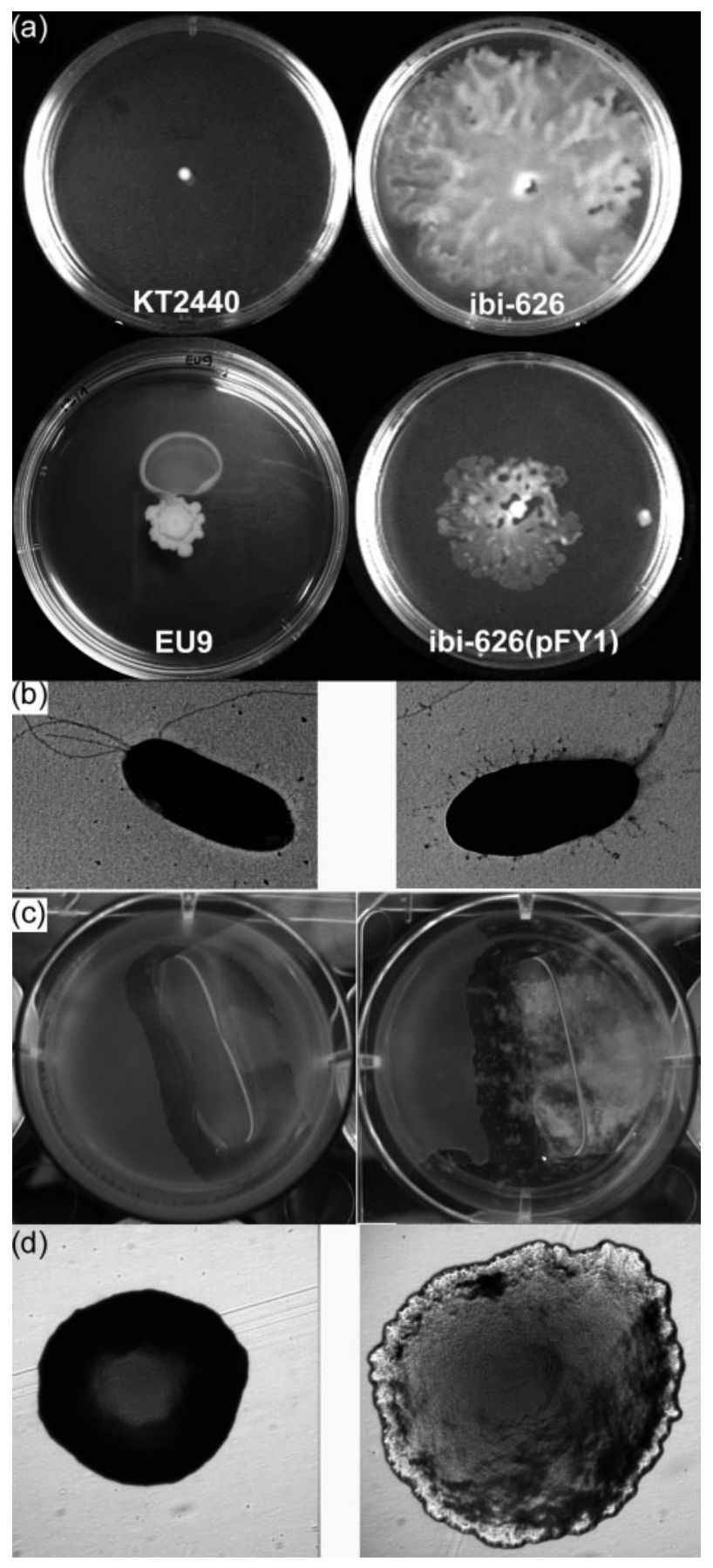

Fig. 5. Phenotype comparison between KT2440 (left) and ibi-626 (right). (a) Swarming motility (intermediate phenotypes caused by the olpA mutation and by olpA in multicopy are also shown); (b) transmission electron microscopy of cells; (c) colony morphology; (d) pellicle formation. 
also disrupted (Gjermansen et al., 2005, 2010). Interestingly, the four olpA mutants identified in the initial screening for ibi clones had the same point mutation in lapG.

The above results indicated that both olpA and lapG mutations contribute to increased adhesion and reduced detachment of ibi-626. The involvement of the lapG mutation in the phenotype of ibi-626 was confirmed by cloning the intact gene from KT2440 into the vector pBBR1-MCS5, and introducing the resulting plasmid, pME164, into KT2440, EU9 and ibi-626. As shown in Fig. 4, the presence of lapG in multicopy inhibited adhesion of all strains to glass.

\section{Pleiotropic effects of lapG and olpA mutations}

In $P$. aeruginosa, biofilm formation and swarming motility are divergently regulated (Kuchma et al., 2010), so that conditions that promote the transition to the sessile lifestyle are associated with an inhibition of swarming motility (Kuchma et al., 2007, 2010; Merritt et al., 2007). However, swarming motility was enhanced in mutant ibi-626 with respect to the wild-type strain (Fig. 5a), suggesting that the two processes are not inversely regulated in $P$. putida. Complementation of the olpA mutation with pFY1 resulted in an intermediate phenotype in terms of swarming (Fig. 5a), and a similar phenotype was observed for mutant EU9, indicating that both mutations (olpA and lapG) contribute to the increase in swarming motility. It has been reported that type IV pili participate in this surface movement in P. putida KT2440 (Matilla et al., 2007). Comparison of KT2440 with ibi-626 by transmission electron microscopy revealed that cells of the ibi-626 mutant had a larger number of surface appendages (presumably pili) than the wild-type (Fig. 5b). This could account for the differences in swarming motility. The mutant also showed altered colony morphology (Fig. 5c) and formed a denser, clumpy pellicle at the air-liquid interface when grown under static conditions (Fig. 5d). The colony morphology and pellicle formation of EU9, however, were indistinguishable from those of KT2440.

We considered the possibility that these two characteristics of ibi-626 were due to an increase in EPS production.
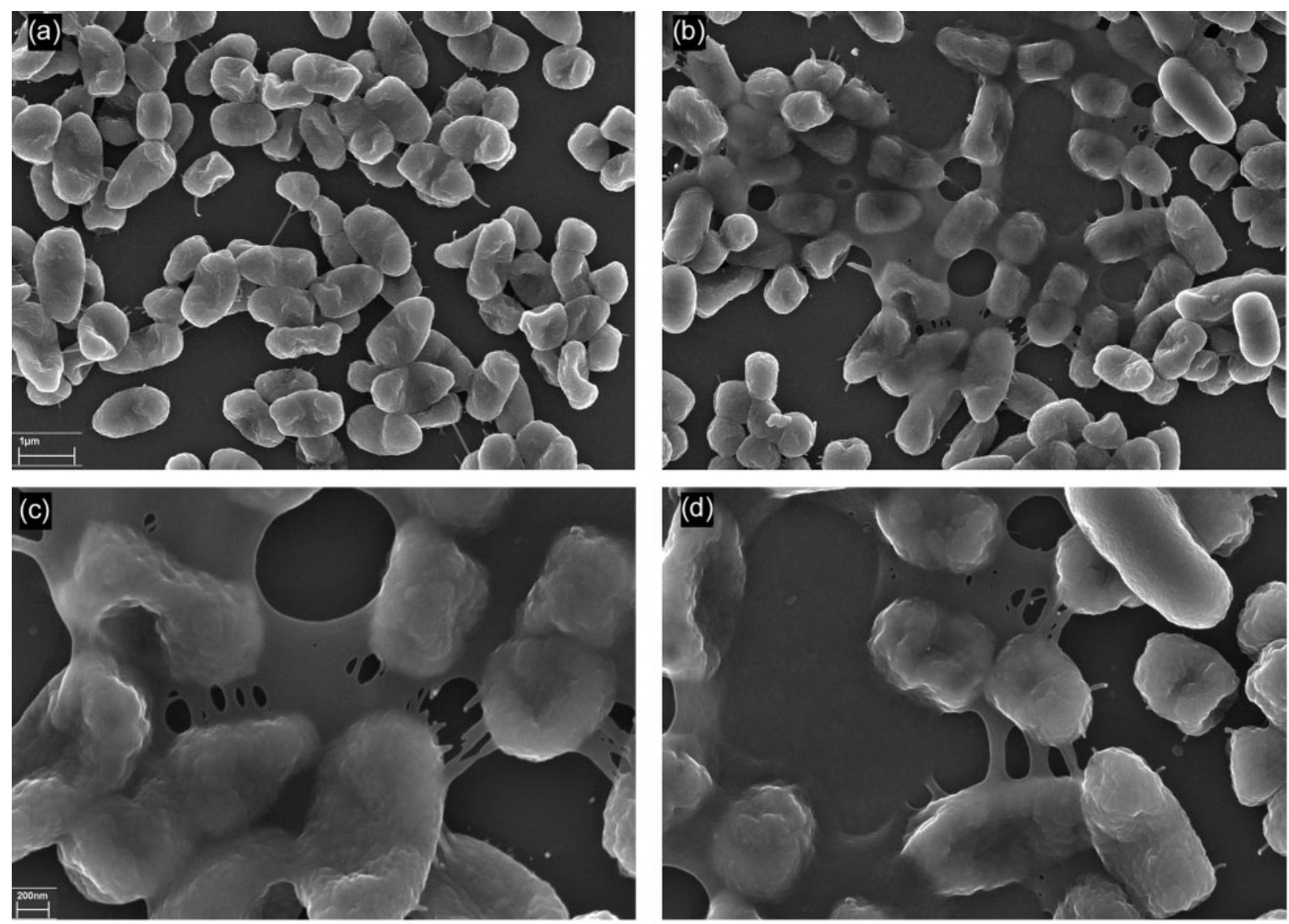

Fig. 6. Scanning electron microscopy images of KT2440 (a) and ibi-626 (b, c, d) cells attached to glass coverslips after 6 h of growth in LB; (c) and (d) correspond to details of (b) at higher magnification. Bars: (a) $1 \mu \mathrm{m}$; (c) $200 \mathrm{~nm}$. 
Scanning electron microscopy was also used to study cells of both strains after $6 \mathrm{~h}$ of biofilm development on glass coverslips. Compact aggregates of ibi-626 cells could be observed, with an extracellular matrix clearly distinguishable, whereas KT2440 cells at this stage were only beginning to form microcolonies and the extracellular matrix was less evident (Fig. 6). Quantification of EPS after dialysis and lyophilization confirmed that the amount of EPS was larger in the ibi-626 mutant than in the wild-type (data not shown). Analysis of total carbohydrates and DNA did not indicate significant differences between the two strains. However, the protein content of the extracellular matrix was 1.7-fold higher in the mutant than in the wild-type (2.62 and $1.59 \mathrm{mg} \mathrm{ml}^{-1}$, respectively).

The robustness of the biofilms formed by KT2440 and ibi626 was tested by analysing biofilm dispersal after enzymic treatments targeting components of the extracellular matrix. As shown in Fig. 7, no significant differences between the two strains were observed after DNase treatment, whereas ibi-626 biofilms were more resistant to protease, requiring prolonged exposure to this enzyme for disaggregation. These and the data above indicate that in ibi-626 there is an increase in the amount of extracellular proteins that form part of the biofilm matrix, suggesting that LapG exerts a similar role in P. putida as that in P. fluorescens. Addition of cellulase did not have a significant effect on biofilms of either strain (data not shown), which is consistent with indications that cellulose is not the most relevant EPS during biofilm formation by $P$. putida under the conditions used here (Nielsen et al., 2011; Nilsson et al., 2011; Matilla et al., 2011).

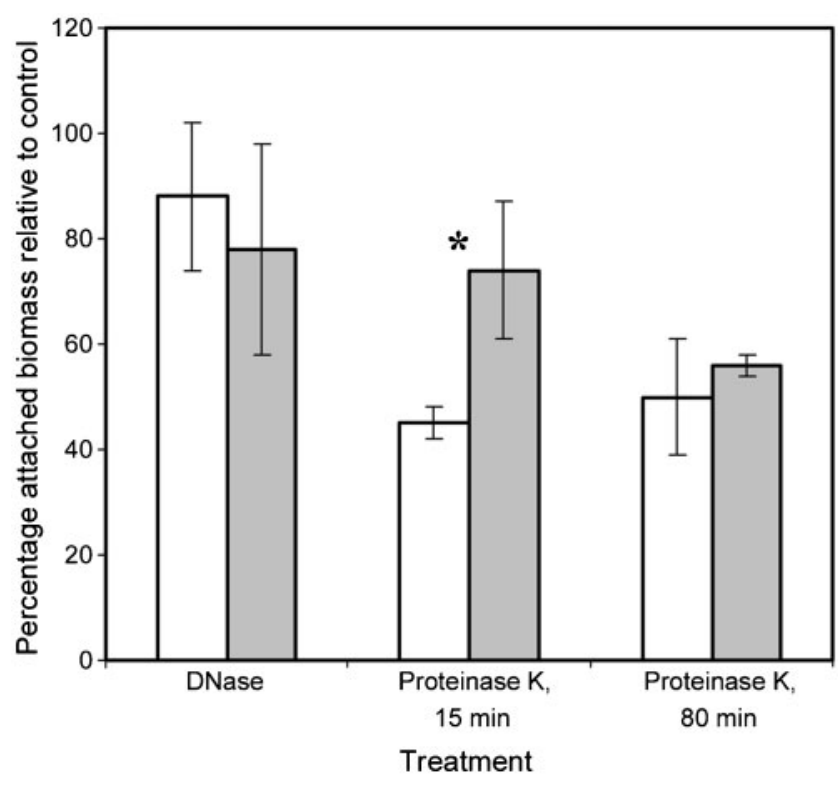

Fig. 7. Dispersal of KT2440 (white bars) and ibi-626 (grey bars) biofilms after treatment with DNase and proteinase $\mathrm{K}$ for 15 or $80 \mathrm{~min}$. Data correspond to mean \pm SD of three experiments. The asterisk indicates a significant difference $(P=0.01)$.

\section{DISCUSSION}

Genetic analysis of biofilm formation has for the most part been approached with 'function loss' strategies, oriented towards the identification of mutations that cause defects in bacterial attachment and/or biofilm development. However, our understanding of how bacteria adapt to the biofilm lifestyle, and the selective forces acting on sessile populations, can also benefit from the analysis of mutations that give rise to variants with increased biofilm formation capacity. This approach may give indications of the potential specialization of a portion of the bacterial population towards a sessile lifestyle, either as the result of bistability or due to phase variation phenomena. Bistability, i.e. the coexistence of two subpopulations that show different gene expression programs within a genetically identical population, has been proposed as a mechanism underlying the specialization of bacteria in biofilms (Chai et al., 2008). In this work we have obtained transposon mutants that show a hyperadherent phenotype and characterized one of them, ibi-626. The dramatic phenotype exhibited by this mutant turned out to be the consequence of the combined effects of two mutations, the disruption of the putative lipoprotein OlpA by the transposon insertion, and an additional point mutation in lapG. The former seems to be responsible for increased early attachment, while the latter contributes to increased biofilm development and prevents detachment of cells from the established biofilm.

The specific role of OlpA remains to be fully explored. Analysis of its sequence reveals the presence of a tetratricopeptide repeat profile. This profile appears in different proteins, among them a family of lipoproteins (InterPro IPR017689) that includes $\mathrm{YfiO}$, involved in outer membrane assembly in E. coli (Gatsos et al., 2008), and ComL, which participates in DNA uptake and transformation in Neisseria gonorrhoeae (Fussenegger et al., 1997). However, OlpA has little sequence similarity with these two proteins, and homologues of OlpA seem to be restricted to Pseudomonas species. Several lipoproteins have been described as having an effect on biofilm formation by different bacteria (Vasseur et al., 2007; Uhlich et al., 2009). In P. putida, expression of the outer membrane lipoprotein NlpD is downregulated following adhesion to the solid surface (Sauer \& Camper, 2001), and recently, NlpI, an E. coli lipoprotein, has been shown to negatively affect production of extracellular DNA (Sánchez-Torres et al., 2010), which in other microorganisms is an important component of the extracellular matrix in biofilms. In P. putida KT2440, however, extracellular DNA seems to have a secondary role, since DNase treatment of biofilms has a limited effect on the biomass associated with the surface, while protease treatment causes significant disruption of the biofilm. The fact that ibi-626 biofilms were more resistant to protease than wild-type biofilms is in agreement with the proteolytic effect of LapG upon LapA (Newell et al., 2011). The absence of LapG would prevent LapA, the major adhesin of $P$. putida, from being cleaved from the bacterial surface and hence would increase the protein content of the biofilm matrix. 
The accumulation of two independent mutations in ibi-626 could be interpreted as an indication that the enrichment method used had favoured the selection of spontaneous lapG mutants present in the planktonic population, while the additional effect of the olpA mutation would have contributed to their being chosen as highly hyperadherent in the analysis of individual clones. Alternatively, the transposon insertion in olpA could be responsible for the increased attachment initiation, the point mutation in lapG being selected for within the biofilm population, as it might confer an advantage under conditions designed to favour sessile growth.

Preliminary data suggest that the second interpretation is correct. An enrichment and selection method identical to that used to identify ibi mutants was employed with wildtype KT2440 cultures. Under the same conditions and with the same number of biofilm enrichment rounds, no hyperadherent variants were obtained. However, when the enrichment rounds were doubled, three, out of 1920, clones that showed increased biofilm formation could be identified. These results are consistent with the selection of spontaneous hyperadherent variants under conditions that favour prolonged sessile growth. In the case of ibi-626, these conditions could have been promoted by the increased early attachment caused by disruption of olpA. Interestingly, sequence analysis of olpA and lap $G$ in the three spontaneous variants showed that in all cases the two ORFs were intact, suggesting that multiple genetic traits can be altered to result in a bias towards the sessile mode of life.

\section{ACKNOWLEDGEMENTS}

We thank J. D. Alché for assistance with confocal and transmission electron microscopy. This work was supported by a grant from the Plan Nacional de I+D + I and FEDER (BFU2007-64270). F. Y.-C. was the recipient of a Formación de Personal Investigador (FPI) fellowship from the Spanish Ministry of Science and Innovation.

\section{REFERENCES}

Altman, E., Brisson, J. R. \& Perry, M. B. (1987). Structure of the capsular polysaccharide of Haemophilus pleuropneumoniae serotype 5. Eur J Biochem 170, 185-192.

Anderson, G. G. \& O'Toole, G. A. (2008). Innate and induced resistance mechanisms of bacterial biofilms. Curr Top Microbiol Immunol 322, 85-105.

Banin, E., Brady, K. M. \& Greenberg, E. P. (2006). Chelator-induced dispersal and killing of Pseudomonas aeruginosa cells in a biofilm. Appl Environ Microbiol 72, 2064-2069.

Campisano, A., Overhage, J. \& Rehm, B. H. (2008). The polyhydroxyalkanoate biosynthesis genes are differentially regulated in planktonicand biofilm-grown Pseudomonas aeruginosa. J Biotechnol 133, 442452.

Cérantola, S., Bounéry, J., Segonds, C., Marty, N. \& Montrozier, H. (2000). Exopolysaccharide production by mucoid and non-mucoid strains of Burkholderia cepacia. FEMS Microbiol Lett 185, 243246.

Chai, Y., Chu, F., Kolter, R. \& Losick, R. (2008). Bistability and biofilm formation in Bacillus subtilis. Mol Microbiol 67, 254-263.
Coenye, T. (2010). Social interactions in the Burkholderia cepacia complex: biofilms and quorum sensing. Future Microbiol 5, 10871099.

de Lorenzo, V., Herrero, M., Jakubzik, U. \& Timmis, K. N. (1990). Mini-Tn 5 transposon derivatives for insertion mutagenesis, promoter probing, and chromosomal insertion of cloned DNA in Gramnegative eubacteria. J Bacteriol 172, 6568-6572.

Dennis, J. J. \& Zylstra, G. J. (1998). Improved antibiotic-resistance cassettes through restriction site elimination using Pfu DNA polymerase PCR. Biotechniques 25, 772-774, 776.

Espinosa-Urgel, M., Salido, A. \& Ramos, J. L. (2000). Genetic analysis of functions involved in adhesion of Pseudomonas putida to seeds. J Bacteriol 182, 2363-2369.

Fernández-Piñar, R., Ramos, J. L., Rodríguez-Herva, J. J. \& Espinosa-Urgel, M. (2008). A two-component regulatory system integrates redox state and population density sensing in Pseudomonas putida. J Bacteriol 190, 7666-7674.

Fussenegger, M., Rudel, T., Barten, R., Ryll, R. \& Meyer, T. F. (1997). Transformation competence and type-4 pilus biogenesis in Neisseria gonorrhoeae - a review. Gene 192, 125-134.

Gatsos, X., Perry, A. J., Anwari, K., Dolezal, P., Wolynec, P. P., Likić, V. A., Purcell, A. W., Buchanan, S. K. \& Lithgow, T. (2008). Protein secretion and outer membrane assembly in Alphaproteobacteria. FEMS Microbiol Rev 32, 995-1009.

Gjermansen, M., Ragas, P., Sternberg, C., Molin, S. \& TolkerNielsen, T. (2005). Characterization of starvation-induced dispersion in Pseudomonas putida biofilms. Environ Microbiol 7, 894-904.

Gjermansen, M., Ragas, P. \& Tolker-Nielsen, T. (2006). Proteins with GGDEF and EAL domains regulate Pseudomonas putida biofilm formation and dispersal. FEMS Microbiol Lett 265, 215-224.

Gjermansen, M., Nilsson, M., Yang, L. \& Tolker-Nielsen, T. (2010). Characterization of starvation-induced dispersion in Pseudomonas putida biofilms: genetic elements and molecular mechanisms. Mol Microbiol 75, 815-826.

Hengge, R. (2009). Principles of c-di-GMP signalling in bacteria. Nat Rev Microbiol 7, 263-273.

Heydorn, A., Nielsen, A. T., Hentzer, M., Sternberg, C., Givskov, M., Ersbøll, B. K. \& Molin, S. (2000). Quantification of biofilm structures by the novel computer program COMSTAT. Microbiology 146, 2395-2407.

Hinsa, S. M., Espinosa-Urgel, M., Ramos, J. L. \& O'Toole, G. A. (2003). Transition from reversible to irreversible attachment during biofilm formation by Pseudomonas fluorescens WCS365 requires an $\mathrm{ABC}$ transporter and a large secreted protein. Mol Microbiol 49, 905918.

Høiby, N., Bjarnsholt, T., Givskov, M., Molin, S. \& Ciofu, O. (2010). Antibiotic resistance of bacterial biofilms. Int J Antimicrob Agents 35, 322-332.

Koch, B., Jensen, L. E. \& Nybroe, O. (2001). A panel of Tn7-based vectors for insertion of the $g f p$ marker gene or for delivery of cloned DNA into Gram-negative bacteria at a neutral chromosomal site. J Microbiol Methods 45, 187-195.

Kovach, M. E., Elzer, P. H., Hill, D. S., Robertson, G. T., Farris, M. A., Roop, R. M., II \& Peterson, K. M. (1995). Four new derivatives of the broad-host-range cloning vector pBBR1MCS, carrying different antibiotic-resistance cassettes. Gene 166, 175-176.

Kuchma, S. L., Brothers, K. M., Merritt, J. H., Liberati, N. T., Ausubel, F. M. \& O'Toole, G. A. (2007). BifA, a cyclic-di-GMP phosphodiesterase, inversely regulates biofilm formation and swarming motility by Pseudomonas aeruginosa PA14. J Bacteriol 189, 8165-8178.

Kuchma, S. L., Ballok, A. E., Merritt, J. H., Hammond, J. H., Lu, W., Rabinowitz, J. D. \& O'Toole, G. A. (2010). Cyclic-di-GMP-mediated 
repression of swarming motility by Pseudomonas aeruginosa: the pilY1 gene and its impact on surface-associated behaviors. J Bacteriol 192, 2950-2964.

Lennox, E. S. (1955). Transduction of linked genetic characters of the host by bacteriophage P1. Virology 1, 190-206.

Matilla, M. A., Ramos, J. L., Duque, E., de Dios Alché, J., EspinosaUrgel, M. \& Ramos-González, M. I. (2007). Temperature and pyoverdine-mediated iron acquisition control surface motility of Pseudomonas putida. Environ Microbiol 9, 1842-1850.

Matilla, M. A., Travieso, M. L., Ramos, J. L. \& Ramos-González, M. I. (2011). Cyclic diguanylate turnover mediated by the sole GGDEF/ EAL response regulator in Pseudomonas putida: its role in the rhizosphere and an analysis of its target processes. Environ Microbiol [Epub ahead of print]. doi:10.1111/j.1462-2920.2011.02499.x.

Merritt, J. H., Brothers, K. M., Kuchma, S. L. \& O'Toole, G. A. (2007). SadC reciprocally influences biofilm formation and swarming motility via modulation of exopolysaccharide production and flagellar function. J Bacteriol 189, 8154-8164.

Monds, R. D. \& O'Toole, G. A. (2009). The developmental model of microbial biofilms: ten years of a paradigm up for review. Trends Microbiol 17, 73-87.

Nelson, K. E., Weinel, C., Paulsen, I. T., Dodson, R. J., Hilbert, H., Martins dos Santos, V. A. P., Fouts, D. E., Gill, S. R., Pop, M. \& other authors (2002). Complete genome sequence and comparative analysis of the metabolically versatile Pseudomonas putida KT2440. Environ Microbiol 4, 799-808.

Newell, P. D., Boyd, C. D., Sondermann, H. \& O'Toole, G. A. (2011). A c-di-GMP effector system controls cell adhesion by inside-out signaling and surface protein cleavage. PLoS Biol 9, e1000587.

Nicolella, C., van Loosdrecht, M. C. \& Heijnen, J. J. (2000). Wastewater treatment with particulate biofilm reactors. J Biotechnol 80, 1-33.

Nielsen, L., Li, X. \& Halverson, L. J. (2011). Cell-cell and cell-surface interactions mediated by cellulose and a novel exopolysaccharide contribute to Pseudomonas putida biofilm formation and fitness under water-limiting conditions. Environ Microbiol 13, 1342-1356.

Nilsson, M., Chiang, W. C., Fazli, M., Gjermansen, M., Givskov, M. \& Tolker-Nielsen, T. (2011). Influence of putative exopolysaccharide genes on Pseudomonas putida KT2440 biofilm stability. Environ Microbiol 13, 1357-1369.

O'Toole, G. A. \& Kolter, R. (1998). Initiation of biofilm formation in Pseudomonas fluorescens WCS365 proceeds via multiple, convergent signalling pathways: a genetic analysis. Mol Microbiol 28, 449-461.

Patriquin, G. M., Banin, E., Gilmour, C., Tuchman, R., Greenberg, E. P. \& Poole, K. (2008). Influence of quorum sensing and iron on twitching motility and biofilm formation in Pseudomonas aeruginosa. J Bacteriol 190, 662-671.

Pham, T. H., Webb, J. S. \& Rehm, B. H. (2004). The role of polyhydroxyalkanoate biosynthesis by Pseudomonas aeruginosa in rhamnolipid and alginate production as well as stress tolerance and biofilm formation. Microbiology 150, 3405-3413.

Regenhardt, D., Heuer, H., Heim, S., Fernandez, D. U., Strömpl, C., Moore, E. R. B. \& Timmis, K. N. (2002). Pedigree and taxonomic credentials of Pseudomonas putida strain KT2440. Environ Microbiol 4, 912-915.

Römling, U. \& Simm, R. (2009). Prevailing concepts of c-di-GMP signaling. Contrib Microbiol 16, 161-181.

Sánchez-Torres, V., Maeda, T. \& Wood, T. K. (2010). Global regulator $\mathrm{H}-\mathrm{NS}$ and lipoprotein NlpI influence production of extracellular DNA in Escherichia coli. Biochem Biophys Res Commun 401, 197-202.

Sauer, K. \& Camper, A. K. (2001). Characterization of phenotypic changes in Pseudomonas putida in response to surface-associated growth. J Bacteriol 183, 6579-6589.

Sauer, K., Camper, A. K., Ehrlich, G. D., Costerton, J. W. \& Davies, D. G. (2002). Pseudomonas aeruginosa displays multiple phenotypes during development as a biofilm. J Bacteriol 184, 1140-1154.

Singh, R., Paul, D. \& Jain, R. K. (2006). Biofilms: implications in bioremediation. Trends Microbiol 14, 389-397.

Sturgill, G., Toutain, C. M., Komperda, J., O'Toole, G. A. \& Rather, P. N. (2004). Role of CysE in production of an extracellular signaling molecule in Providencia stuartii and Escherichia coli: loss of CysE enhances biofilm formation in Escherichia coli. J Bacteriol 186, 76107617.

Terada, M., Kuroda, T., Matsuyama, S. I. \& Tokuda, H. (2001). Lipoprotein sorting signals evaluated as the LolA-dependent release of lipoproteins from the cytoplasmic membrane of Escherichia coli. J Biol Chem 276, 47690-47694.

Toutain, C. M., Caizza, N. C., Zegans, M. E. \& O'Toole, G. A. (2007). Roles for flagellar stators in biofilm formation by Pseudomonas aeruginosa. Res Microbiol 158, 471-477.

Ueda, A. \& Wood, T. K. (2009). Connecting quorum sensing, c-diGMP, Pel polysaccharide, and biofilm formation in Pseudomonas aeruginosa through tyrosine phosphatase TpbA (PA3885). PLoS Pathog 5, e1000483.

Uhlich, G. A., Gunther, N. W., IV, Bayles, D. O. \& Mosier, D. A. (2009). The CsgA and Lpp proteins of an Escherichia coli O157:H7 strain affect HEp-2 cell invasion, motility, and biofilm formation. Infect Immun 77, 1543-1552.

Vasseur, P., Soscia, C., Voulhoux, R. \& Filloux, A. (2007). PelC is a Pseudomonas aeruginosa outer membrane lipoprotein of the OMA family of proteins involved in exopolysaccharide transport. Biochimie 89, 903-915.

Vilchez, S., Molina, L., Ramos, C. \& Ramos, J. L. (2000). Proline catabolism by Pseudomonas putida: cloning, characterization, and expression of the put genes in the presence of root exudates. J Bacteriol 182, 91-99.

Yamaguchi, K., Yu, F. \& Inouye, M. (1988). A single amino acid determinant of the membrane localization of lipoproteins in E. coli. Cell 53, 423-432.

Yousef-Coronado, F., Travieso, M. L. \& Espinosa-Urgel, M. (2008). Different, overlapping mechanisms for colonization of abiotic and plant surfaces by Pseudomonas putida. FEMS Microbiol Lett 288, 118124.

Edited by: R. Palmer 